\title{
Conflits Politiques En Côte D'ivoire : Les Facteurs D'emergence Et Cartographie Des Foyers
}

\author{
Constance Boka Abeto, \\ Dakouri Guissa Desmos Francis, \\ Maitre-Assistant, Institut de Géographie Tropicale, \\ Université Félix Houphouët Boigny, Côte d'Ivoire
}

Doi:10.19044/esj.2020.v16n20p176 URL:http://dx.doi.org/10.19044/esj.2020.v16n20p176

\section{Résumé}

Ancienne colonie française, la Côte d'Ivoire était considérée comme un pays prospère et paisible. Toutefois, à la mort du président Félix Houphouët Boigny, la guerre de succession enclenchée par ses héritiers ont attisé des foyers tensions à travers tout le pays. L'objectif principal de cette étude est de mettre en évidence les facteurs de l'émergence de ces foyers de tension et de les cartographier par la suite. L'appareillage méthodologique pour y arriver a fait recours à la revue bibliographique et une enquête de terrain. Les résultats obtenus indiquent que si le tribalisme, la religion, la pauvreté, l'analphabétisme, la densité et l'extrême jeunesse de la population sont autant de facteurs de l'émergence des conflits politiques, c'est toute la zone sud jusqu'au littoral et les régions de "grand pont", Tonkpi, Haut-Sassandra, Bélier, Gbêkê, Bafing, Worodougou et Poro qui en constituent les grands foyers.

Mots-clés: Conflit, Politique, Facteur, Foyer, Côte d'Ivoire 


\title{
Political Conflicts in Cote D'ivoire: Emergence Factors and Home Mapping
}

\author{
Constance Boka Abeto, \\ Dakouri Guissa Desmos Francis, \\ Maitre-Assistant, Institut de Géographie Tropicale, \\ Université Félix Houphouët Boigny, Côte d'Ivoire
}

\begin{abstract}
A former French colony, Côte d'Ivoire, was considered a prosperous and peaceful country. At the death of President Félix Houphouët Boigny, the war of succession initiated by his heirs fueled tensions throughout the country. This paper focuses on highlighting the factors of the emergence of these focal points and in mapping them later. The methodological equipment used to achieve this was the bibliographic review and a field survey. The results obtained indicate that if tribalism, religion, poverty, illiteracy, the density and extreme youth of the population are all factors of the emergence of political conflicts, then the whole southern zone up to the coast and the regions of "Grand Pont", Tonkpi, "Haut-Sassandra", Bélier, Gbêkê, Bafing, Worodougou, and Poro are the major centres.
\end{abstract}

Keywords: Conflict, Politics, Postman, Home, Côte d'Ivoire

\section{Introduction}

Devenue indépendante le 07 août 1960, la Côte d'Ivoire a connu une embellie économique due à une relative stabilité politique. Ce qui a fait d'elle autrefois la vitrine de l'Afrique de l'ouest. Cependant, trente ans après l'indépendance et avec le décès du président Félix Houphouët-Boigny en 1993, une nouvelle ère s'ouvre dans le pays. Les Ivoiriens découvrent véritablement le multipartisme et les tumultes de la démocratie. Ainsi comme l'affirme Koui, (2010, p.37) "Le peuple de Côte d'Ivoire, après la mort du président Houphouët, a été confronté aux affres de la compétition fratricide entre ses politiciens pour s'approprier en exclusivité le pouvoir vacant. En ont résulté des massacres et une guerre civile dont les populations ont payé le plus lourd tribut». En effet, le décès de Félix Houphouët Boigny, premier président de la Côte d'Ivoire indépendante, plonge le pays dans une instabilité chronique ponctuée de fréquentes crises et leurs corollaires de violences. Les ambitions personnelles des hommes politiques et la guerre de succession au 
sein du Parti Démocratique de Côte d'Ivoire (PDCI) au pouvoir, entraîne le pays dès 1993 dans trois décennies de crise.

Les institutions de la république sont éprouvées et la vie politique fortement perturbée par les partis d'opposition, aboutissent au premier coup d'Etat militaire dans l'histoire de la Côte d'Ivoire en décembre 1999 (Bamba, 2015 , p.2). Ce renversement de régime entraînera une longue période d'instabilité politique, couronnée par la crise post-électorale de 2011. Dans ce contexte où les conflits politiques jalonnent l'histoire récente de la Côte d'Ivoire, cette étude tente d'identifier les facteurs de leur émergence et de mettre en évidence à partir de la cartographie les zones les plus touchées. La réponse à cette préoccupation constitue l'intérêt et l'objectif principal de cet article. Cependant, la pertinence scientifique d'un tel travail nécessite une brève revue de recherche pour faire l'état des lieux sur la question posée.

\section{1- $\quad$ Etat de la question}

De nombreux écrits existent sur les conflits et leurs caractéristiques. En effet, Etymologiquement, le terme conflit vient du latin « conflictus » qui signifie heurt, choc. Le conflit peut se définir comme une situation impliquant un différend ou une divergence d'opinions dans lequel des valeurs, des besoins et/ou des intérêts (tangibles) s'affrontent. Le conflit est généralement perçu à la façon d'un cycle, susceptible d'évoluer vers une situation violente, mais pas nécessairement (Soriano, 2001, p.5).

$\mathrm{Au}$ niveau géographique, le conflit mêle peu ou prou le couple représentation de l'espace/espace des représentations selon des dosages variables. Donc ce qui importe le plus, est d'imposer non pas son usage, ni d'affirmer sa légitimité, mais bien d'amener tous les acteurs concurrents sur son propre terrain, d'imposer son espace de représentation donc les règles du jeu spatial (Retaillé, 2011, p.9). Le conflit diffère donc de la compétition, de la concurrence, du duel, etc et est essentiellement d'ordres stratégique et politique.

Les conflits politiques opposent donc des groupes au sens le plus général du terme. Ils opposent des partis politiques, des États, des alliances d'États, aussi bien que des classes sociales, des groupes professionnels, des nations ou des nationalités, des communautés de langue ou de religion différentes. Dans tous les cas, un conflit politique concerne des groupes plus ou moins structurés et institutionnalisés (Canivez, 2008, p.164).

L'entrée en conflit de ces groupes est due au fait que les intérêts de ceux-ci sont divergents. En réalité, un intérêt est tout ce pour quoi un groupe est prêt à entrer en conflit, qu'il s'agisse des intérêts économiques d'une classe sociale, des intérêts culturels d'une minorité ethnique, des intérêts stratégiques d'un État, etc. Les groupes s'efforcent de montrer que leurs intérêts sont légitimes, de même que les moyens utilisés (violents ou non violents) pour les 
faire valoir. De ce point de vue, on peut dire que les conflits politiques sont des conflits pour la reconnaissance (Canivez, 2008, p.165). Ainsi, la cristallisation identitaire et l'exaspération des différences qui engendrent le conflit fonctionnent sur le registre complémentaire de « l'ethnicité morale » et $\mathrm{du}$ «tribalisme politique» (Lonsdale, 2003, p.36) qui respectivement définissent le «nous » et le «eux», dans un mouvement d'intégration/exclusion complémentaire (Calas, 2011, p.299).

Parlant des variables contextuelles, F. Joerin et al. (2005, pp.328-329) indiquent que la densité de la population, le niveau d'éducation, l'âge du bâti, la densité de commerce de quartier, le niveau de revenu, le niveau de disponibilité, les horaires professionnelles et le type de milieu familial sont autant de facteurs de l'émergence des conflits urbain au Québec.

De façon générale, Cette brève revue a montré que les conflits sont des hostilités, des sources de contestations, d'antagonisme, d'opposition, de lutte armée, et heurts entre les communautés, les peuples et les Etats. Cependant, cette revue reste généraliste et ne traite pas de façon spécifique les conflits politiques en Côte d'Ivoire encore moins de la distribution spatiale des foyers de ceux-ci. C'est ainsi que cet article trouve toute sa place dans la mesure où le travail ici va essayer d'identifier les facteurs de l'émergence et les grands foyers de ces conflits dans le pays.

\section{2- $\quad$ Matériels et méthode}

\section{1- $\quad$ Aspect synthétique de la Côte d'Ivoire}

La Côte d'Ivoire est un pays situé en Afrique de l'ouest entre le $5^{\text {ème }}$ et $10^{\text {ème }}$ degré, dans l'hémisphère nord, entre le tropique du Cancer et l'Equateur. Elle est délimitée au nord par le Burkina-Faso et le Mali. A l'ouest, par le Libéria et la Guinée, à l'est par le Ghana et est bordée au sud par l'océan Atlantique (figure 1). Sa superficie est de $322.462 \mathrm{~km} 2$, pour une population d'environ 22671331 habitants selon l'Institut National Statistique (INS) en 2014. 


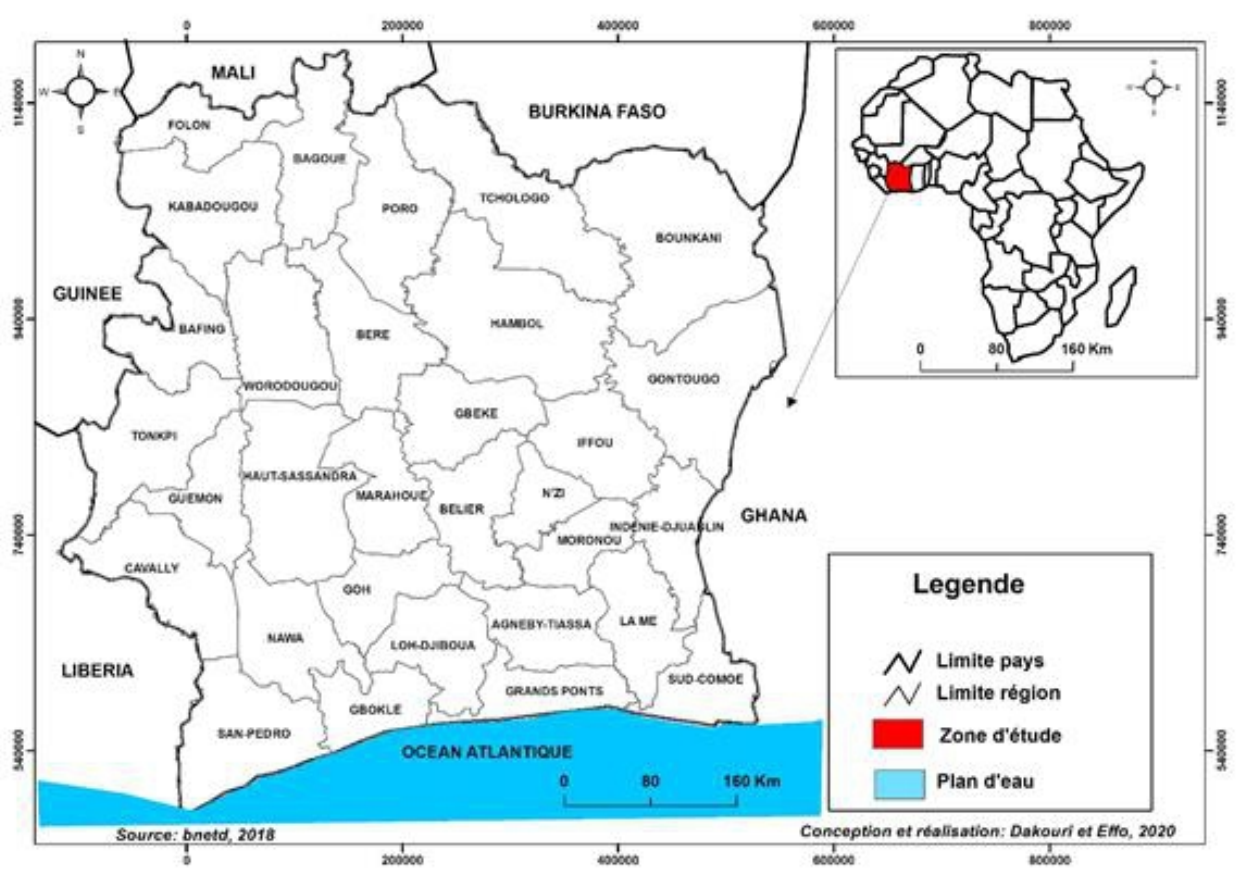

Figure 1. Localisation de la Côte d'Ivoire

La Côte d'Ivoire, qui dispose de solides atouts économiques, est une puissance sous régionale. Elle possède des infrastructures héritées des deux décennies du «miracle ivoirien» (1960-1980): 2e port d'Afrique subsaharienne, important réseau routier, aéroport international récemment agrandi. Dans le secteur agricole le pays est le 1er producteur de cacao au monde avec plus de 2,2 millions de tonnes en 2019. Il figure aux tout premiers rangs africains pour plusieurs autres productions agricoles d'exportation (caoutchouc, noix de cajou, coton, café, palmier à huile, banane, ananas, cola). Le secteur secondaire est dominé par le raffinage de pétrole brut, le Batiment et Travaux Publiques (BTP) et la transformation agroalimentaire. Le secteur tertiaire (47\% du PIB) est fortement dominé par les activités bancaires, les transports, la distribution ainsi que les Technologies de l'Information et de la Communication (TIC), dont la téléphonie mobile (trois opérateurs). Le pays assure son autosuffisance énergétique grâce à l'exploitation depuis quelques années de gisements de gaz et de pétrole; ils lui ont permis d'exporter de l'électricité et des produits pétroliers dans la sous-région.

\section{2- Technique de collecte des données}

L'appareillage méthodologique retenu pour cette étude se résume en trois étapes fondamentales que sont : la recherche documentaire, l'enquête de terrain à travers l'administration d'un questionnaire et le traitement des données. La recherche bibliographique offre un regard global et théorique sur 
le sujet a permis d'identifier différents types de conflits, de cerner les causes et les solutions apportées.

Pour rester dans le cadre des conflits politiques, la sélection des personnes interrogées s'est faite au sein des militants des principaux partis politiques à savoir le Rassemblement Des Républicains (RDR), le Front Populaire Ivoirien (FPI) et le Parti Démocratique de Côte d'Ivoire (PDCI). Elle a également tenu compte du type d'autorités politiques et administratives (Maires, Député, Préfets, Sous-préfets, chefs de villages et leurs notables). Ces personnes ont été visitées et interrogées de Mars à Mai 2019 par notre équipe d'enquête. La taille de l'échantillon a été calculé manuellement à l'aide de la formule de Christensen Howard suivante :

$\mathrm{n}=\mathrm{t}^{2} \times \mathrm{p} \times(1-\mathrm{p}) / \mathrm{m}^{2}$

- $\mathrm{n}$ : Taille d'échantillon minimale

- $\mathrm{t}$ : Niveau de confiance (la valeur type du niveau de confiance de $95 \%$ sera 1,96 )

- $\quad$ p : Ecart type de la population (8) selon l'Institut National de la Statistique (INS)

- $\mathrm{m}$ : Marge d'erreur $(1,5)$

En application numérique, on obtient ceci :

$\mathrm{n}=1,96^{2} \times 8^{2} / 1,5^{2}=109,2721$ soit 110 individus

La taille minimale requise pour l'échantillon est donc de 110 individus enquêtés dans chaque ville. Au total, 770 personnes ont constitué l'échantillon à choix raisonné à partir de critères jugés pertinents. Il s'agit des critères comme être militant de parti politique, être résident dans la localité, être un élu de la localité, être responsable administratif (sous-préfet, préfet, chef de village) de la localité. Elles ont été interrogées dans les villes suivantes: Abidjan, Bouaké, Korhogo, Man, San-Pedro, Gagnoa et Abengourou sélectionnées également par choix raisonné à partir des critères tels que : être chef-lieu de région, avoir été au moins une fois théâtre de violences politiques. Le dépouillement des données a permis d'aboutir aux résultats suivants.

\section{3- Résultats et discussion}

\section{1- Les facteurs d'émergence des conflits politiques}

Les facteurs d'émergence des conflits politiques identifiés au terme de l'enquête de terrain sont : la densité de la population, le tribalisme, le niveau d'instruction, la réligion, la paupérisation de la population et l'extrême jeunesse de la population. Il ressort de l'analyse de Figure 2 que les facteurs dominant pour l'émergence des conflits politiques sont par ordre d'importance 
le tribalisme (32\%), la réligion (30\%), et la paupérisation de la population $(17 \%)$.
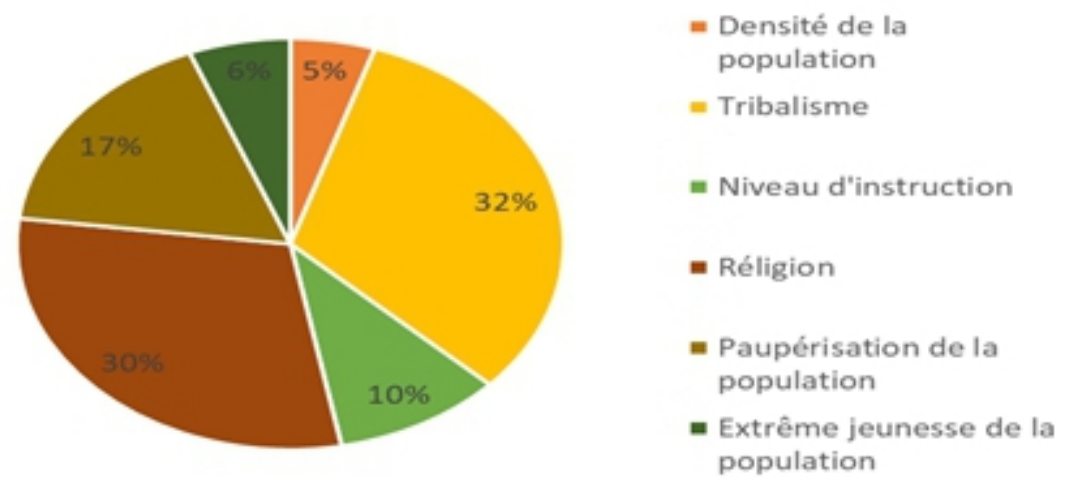

Figure 2. Les déterminants socio-économiques des conflits politiques en Côte d'Ivoire.

Source: notre enquête de terrain, 2019

Ces déterminants socio-économiques des conflits politiques retenus pour cette étude peuvent s'expliquer de la manière suivante.

\subsection{1- Une population galopante comme source d'activité conflictuelle}

La culture du café et du cacao, l'exploitation forestière et la construction des routes exigeaient une forte main d'œuvre. Cela a donc entrainé un vaste mouvement migratoire de toute la sous-région vers la Côted'Ivoire. C'est ainsi que beaucoup de ressortissants des pays comme la Haute Volta (actuel Burkina Faso), le Soudan (actuel Mali), la Guinée, le Sénégal, la Mauritanie, la Syrie, le Liban, le Niger et la France, se sont installées en Côted'Ivoire. L'embellie économique qualifiée de " miracle ivoirien » qui a marqué les deux premières décennies de l'indépendance du pays s'est accompagné d'une immigration de plus en plus forte. Ainsi, la population de la Côte d'Ivoire a été multipliée par 3,4 entre 1975 et 2014 selon l'INS.

Or, le nombre de personnes donnée, (pour évoquer le facteur "densité de la population"), qui occupent une portion bien précise d'un milieu, d'un territoire peut être source de conflits lorsque se rarefient les bien à se partager. Celles-ci entrent donc en conflit du fait de leurs intérêts économiques, culturels et stratégiques opposés. Ce qui fait dire à Joerin et al. $(2005$, p. 328) que l'activité conflictuelle est plus intense dans les secteurs les plus densément peuplés. Ainsi, une forte identité favoriserait l'émergence d'une conflictualité et en retour, celle-ci renforcerait le mouvement identitaire (Lecourt \& Baudelle, 2004, p. 11). 


\subsection{2- Le tribalisme comme source d'activité conflictuelle}

On peut définir le tribalisme comme étant une manifestation qui prend pour base le groupe ethnique. C'est la cause la plus apparente avec 32\% des enquêtés. En effet, à la disparition du président Houphouët-Boigny, plusieurs prétendants au poste de président de la république vont s'affronter : Henri Konan Bédié, l'héritier constitutionnel, Alassane Dramane Ouattara, le Premier ministre qui a assuré l'intérim du président malade et Laurent Gbagbo, le principal opposant.

Henri Konan Bédié est, comme Houphouët-Boigny, de l'ethnie Baoulé, branche du groupe ethnoculturel Akan. Il est donc celui qui pourrait perpétuer le pouvoir Baoulé.

Alassane Dramane Ouattara se réclamant descendant de l'empereur Sékou Ouattara, fondateur de l'empire de Kong, revendique le pouvoir au nom des populations du nord de la Côte-d'Ivoire qui appartiennent aux groupes ethnoculturel Gour (ou Voltaïque) et Mandé.

Laurent Gbagbo est de l'ethnie Bété ; il appartient donc au groupe ethnoculturel Krou situé au sud-ouest de la Côte d'Ivoire. Les populations Krou se reconnaissent dans le combat de Laurent Gbagbo à cause de certaines injustices subies sous le régime de Houphouët-Boigny.

C'est ainsi que les populations qui n'ont pas de formation politique, ont choisi, le plus souvent, de soutenir le leader de leur région, de leur ethnie. Bédié est le mentor des Baoulé (au centre), Alassane Dramane Ouattara celui des Gour et Mandé (au nord), et Gbagbo celui des Krou (au sud-ouest). Parce que le lien tribal est le plus sensible et le plus immédiatement saisissable, il est couramment utilisé par ces politiciens pour faire accepter certains changements. La force représentée par certaines ethnies a servi et sert encore de base aux leaders politiques et aux partis. Ce qui fait dire Bonzon (1967, p. 864) que lorsque des partis se sont créés et lorsque des leaders ont cherché à réunir sous leur nom le maximum de partisans, ils ont d'abord fait appel à leur ethnie ou, plus exactement, lorsqu'elles existaient, aux associations tribales. Or, là où il y a tribalisme, la religion s'y invite.

\subsection{3- La religion comme source d'activité conflictuelle}

En ce qui concerne la religion, il importe de savoir qu'en Afrique, le Christianisme et l'Islam sont les deux principales religions révélées qui génèrent des conflits identitaires. La question religieuse en Afrique pose un certain nombre de problèmes relatifs à l'identité et à l'instrumentalisation religieuse à des fins politiques surtout avec les indépendances dans la plupart des pays africains. Dans cette optique, Huntington (1993, p.12) prédisait un changement de situation. Il soutient que : "après l'effondrement de l'Union soviétique, les motifs d'affrontements et de guerre ne seront plus bien sûr des 
motifs Est-Ouest, mais des affrontements à venir entre grandes zones culturelles, entre grandes aires religieuses ». Depuis vingt ans, les conflits interconfessionnels se sont multipliés en Afrique. Celui entre le nord du Soudan, musulman, et son sud chrétien, a abouti à l'indépendance du Sud Soudan. Les affrontements armés récurrents entre Seleka (musulman) et Anti-Balaka (chrétiens et animistes) en Centrafrique. Au Nigeria, où la population est pour moitié musulmane et pour l'autre chrétienne, les deux communautés se déchirent régulièrement et une secte islamiste Boko Haram, multiplie les attentats. Au Sahel, les intégristes algériens liés à Al Qaïda, de grandes familles Touaregs et des musulmans fanatiques, ont tissé des alliances. Cela montre très clairement que la religion demeure foncièrement une cause de conflits. En Côte d'Ivoire, deux grandes obédiences religieuses se répartissent la population; il s'agit de l'Islam qui concentre $42 \%$ et le Christianisme (34\%) de la population totale résidente du pays. On note par ailleurs que parmi la population étrangère, les musulmans représentent $72 \%$ (contre $18 \%$ de chrétiens de cette population) alors que dans la population ivoirienne, ce sont les chrétiens qui viennent en tête avec $39 \%$ et les musulmans représentent 30\% selon l'INS en 2014. Cette réalité religieuse a été exploitée par des politiciens véreux qui voulaient à tout prix accéder au pouvoir d'Etat. La fibre religieuse utilisée comme prétexte a permis à ces politiciens d'avoir le soutien indéfectible des populations du nord musulman et faire croire à la communauté internationale en 2002 que la guerre ivoirienne était interreligieuse (musulman contre chrétien). Heureusement, les hommes de Dieu, musulmans comme chrétiens y compris bouddhistes et autres, ont créé un forum pour apporter un démenti à cette allégation.

\subsection{4- Le niveau analphatisme comme source d'activité conflictuelle}

L'éducation participe de la modification du comportement humain. Elle est susceptible de faire ressortir son incidence sur le comportement et la compréhension des enjeux comme la démocratie, la paix, etc. C'est pour quoi Horace (1883) soutient que lorsqu'on établit des écoles, l'ignorance, le crime et la misère sont chassés ; les haines diminuent et on fait la fortune et la grandeur du pays par l'aisance, la moralité et le bonheur de chacun.

Malheureusement pour la Côte d'Ivoire, le niveau de l'analphabétisme reste encore à un niveau relativement élevé (63\%) bien qu'ayant enregistré une baisse de 0,5 point en moyenne par an depuis 1998. Les résultats du Recensement Général de la Population et de l'Habitat (RGPH) de 2014 indique que sur la base de la population de 15 ans et plus (13 185520 individus), plus de la moitié $(56,1 \%)$ ne savent ni lire, ni écrire dans une langue quelconque. Ces analphabètes manipulables à souhait par les politiciens se réfêrent à leur communauté ethnique ou religieuse pour leurs choix politiques. 


\subsection{5- La jeunesse de la population comme source d'activité conflictuelle}

Relativement à la structure par âge, la population de la Côte d'Ivoire selon les résultats du RGPH 2014 demeure encore très jeune. Les enfants ( 0 14 ans révolus) au nombre de 9481351 individus représentent $41,8 \%$ de la population totale et les Jeunes (15-34 ans révolus) au nombre de 8048341 individus constituent $35,5 \%$ de la population totale. Ainsi, $77,3 \%$ de la population totale, soit un peu plus 3 personnes sur 4 personnes ont moins de 35 ans. Or, à cet âge, l'homme connaît une hyperactivité, une impulsivité et une maitrise insuffisante de soi. C'est pourquoi l'extrême jeunesse de la population peut être source de conflit si elle n'est pas bien encadrée. Par ailleurs, expliquant les raisons de la prolifération d'enfants soldats dans les crises africaines Ebodé (2006, p.115) souligne que «Pour les belligérants, en effet, le recours aux enfants présente trois principaux avantages d'ordre économique, sociologique et psychologique. Sur le plan économique, les enfants soldats coûtent nettement moins cher. D'un point de sociologique, ces enfants n'ont pas de contraintes familiales. Psychologiquement parlant, enfin, les enfants sont réputés savoir mieux survivre dans la brousse, savoir mieux garder le silence (souffrir en silence) et se montrer rigoureux dans l'application des ordres ». Dans son rapport publié en 2001, l'ONU a ainsi relevé qu'au moins 300000 enfants étaient impliqués dans les conflits, dans une trentaine de pays, dont 120000 pour la seule Afrique au sud du Sahara. (ONU, 2004, p. 3).

\subsection{6- La pauvreté comme source d'activité conflictuelle}

Concernant la paupérisation, nombreux sont les pays africains qui, n'ayant pu profiter de leurs ressources naturelles et humaines ou de leur position géostratégique favorable aux transactions de tout genre, ont sombré dans une situation de pauvreté générant des germes de conflictualité. Ainsi, à partir des années quatre-vingt, le système économique de la Côte d'Ivoire, basé sur l'exportation des produits bruts agricoles, fait apparaître ses limites. Le prix du café et du cacao a connu une baisse drastique. Les produits des paysans étaient achetés à vil prix. Le retournement de la conjoncture économique internationale a plongé le pays dans des difficultés de tous ordres. La demande sociale grandissait, sous la pression démographique, tandis que les terres cultivables se réduisaient. Pour remédier à la crise économique, les institutions de Bretton Woods ont imposé à la Côte d'Ivoire un programme d'ajustement structurel, c'est-à-dire la privatisation des sociétés d'Etat, le dégraissage de la fonction publique, l'arrêt des investissements sociaux, la diminution et le blocage des salaires. Les frustrations étaient donc au sein de toutes les couches sociales. Des groupes vulnérables se sont constitués. Dans 
une telle situation, le risque de semer les germes de la haine et de l'adoption d'une culture de violence est élevé.

\section{2- Une kyrielle de conflits politiques en Côte d'Ivoire}

Joerin et al. (2005, p.325) définissent un conflit comme étant un événement circonscrit dans l'espace et dans le temps (il a un début et une fin), impliquant des protagonistes (acteurs) qui formulent des propositions ou y font objection. Afin d'illustrer cette notion, voici résumés quelques exemples de conflits politiques qui ont eu lieu en Côte d'Ivoire dans le tableau 1 suivant.

Tableau 1. Inventaire des conflits politiques en Côte d'Ivoire

\begin{tabular}{|l|l|}
\hline DATE & LIBELLE DU CONFLIT \\
\hline Août 1970 & Affaire Kragbé Gnangbé (Gagnoa) \\
\hline $\mathbf{1 9 9 0}$ & Mouvements sociaux dans tout le pays \\
\hline $\mathbf{1 9 9 1}$ & Expéditions meurtrières dans les cités universitaires \\
\hline $\mathbf{1 8}$ février 1992 & $\begin{array}{l}\text { Marche du FPI à Abidjan ayant occasionné l'arrestation de plusieurs } \\
\text { opposants }\end{array}$ \\
\hline Octobre 1995 & Boycott actif des élections présidentielles par le FPI et le RDR \\
\hline $\mathbf{2 4}$ décembre 1999 & $\begin{array}{l}\text { Une mutinerie de soldats se transforme en coup d'état à Abidjan. Mise } \\
\text { en place d'un Comité National de Salut Public (CNSP) }\end{array}$ \\
\hline $\mathbf{2 6}$ octobre 2000 & $\begin{array}{l}\text { Violent affrontement entre les partisans du RDR et ceux du FPI à } \\
\text { Abidjan. Ils font plus d'une centaine de morts }\end{array}$ \\
\hline $\mathbf{4}$ décembre 2000 & $\begin{array}{l}\text { Une manifestation du RDR dégénère, entrainant 48 heures de troubles } \\
\text { soldés par une vingtaine de morts à Abidjan. }\end{array}$ \\
\hline $\mathbf{7 - 8}$ Janvier 2001 & Coup d'Etat manqué baptisé "complot de la Mercédès noire" \\
\hline 25 mars 2004 & Coup d'Etat manqué mué en rébellion qui a divisé le pays en deux \\
\hline $\mathbf{4}$ novembre 2004 & Marche insurrectionnelle du RDR à Abidjan \\
\hline 6 novembre 2004 & Tentative de libération des zones sous contrôle de la rébellion \\
\hline $\begin{array}{l}\text { Neuf soldats français seraient tués et une trentaine de blessées dans un } \\
\text { avril 2011 }\end{array}$ & $\begin{array}{l}\text { cantonnement militaire français à Bouaké lors d'une attaque aérienne } \\
\text { gouvernementale. Paris ordonne la destruction des moyens aériens } \\
\text { militaires ivoirienne. La riposte des forces françaises est suivie de } \\
\text { violences anti-françaises }\end{array}$ \\
\hline mai 2006 & $\begin{array}{l}\text { Règlements de conflits fonciers et ethniques qui se transforment en } \\
\text { affrontements entre les partisans de partis politiques RDR et FPI et font } \\
\text { 15 blessés graves à San Pedro. }\end{array}$ \\
$\begin{array}{l}\text { Une tentative de réconciliation entre les autochtones et les allogènes } \\
\text { dégénère en affrontements entre partisans de partis politiques opposés } \\
\text { à Bangolo faisant un mort et 5 blessés graves }\end{array}$ \\
$\begin{array}{l}\text { Crise post-électorale caractérisée par les bombardement de l'armée } \\
\text { française, la force onusienne et les attaques des forces rebelles contre } \\
\text { le régime de Laurent Gbagbo }\end{array}$ \\
\hline
\end{tabular}

Source : Nos enquêtes 2019 


\section{3- Analyse spatiale des conflits politiques}

\subsection{1- Au niveau des régions}

Depuis 1970 beaucoup de conflits politiques et non des moindres ont jalonné l'histoire de la Côte d'Ivoire. Ces conflits qui se sont plus intensifiés entre 1990 et 2011, se sont surtout concentrées à Abidjan, et ses environs. En effet, les zones du sud jusqu'au littoral c'est-à-dire les régions des "Grands Ponts", du Bas Sassandra et du Sud Comoé enregistrent 75\% de ces crises selon la Figure 3. Les 25\% restant sont reparties entre les régions de la vallée de Bandama, des Grands lacs, du Moyen Cavally, du Haut Sassandra, du Worodongou, du Bafing et du Poro.

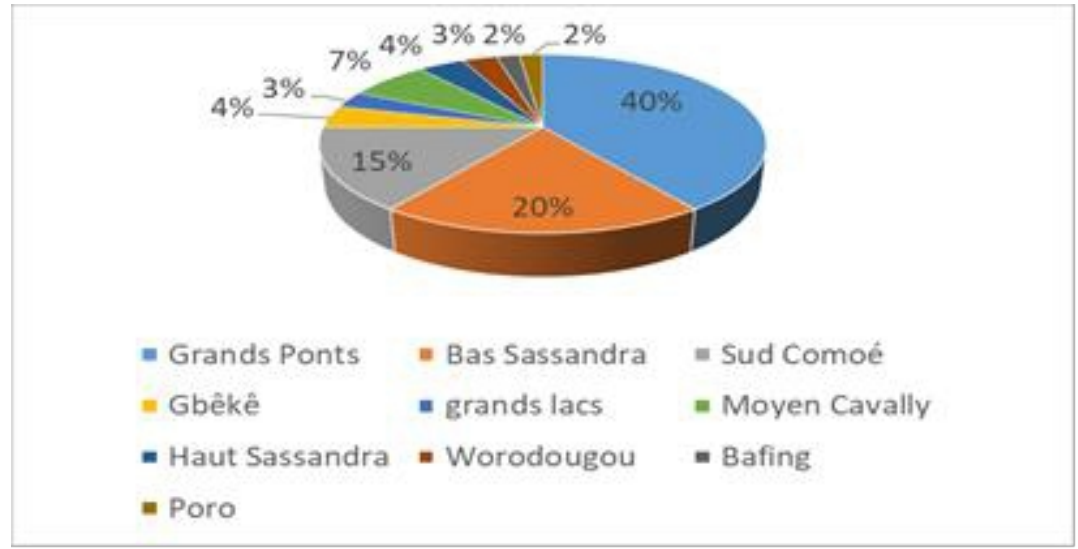

Figure 3. Répartition des conflits selon les régions

Source : Nos enquêtes, 2019

En effet, cette zone qui s'étend sur sept régions administratives telles que Tonkpi, Haut-Sassandra, Bélier, Gbêkê, Bafing, Worodougou et Poro (figure 4) constitue l'avenir économique du pays, grâce à la présence de ressources forestières, agricoles et minières. 


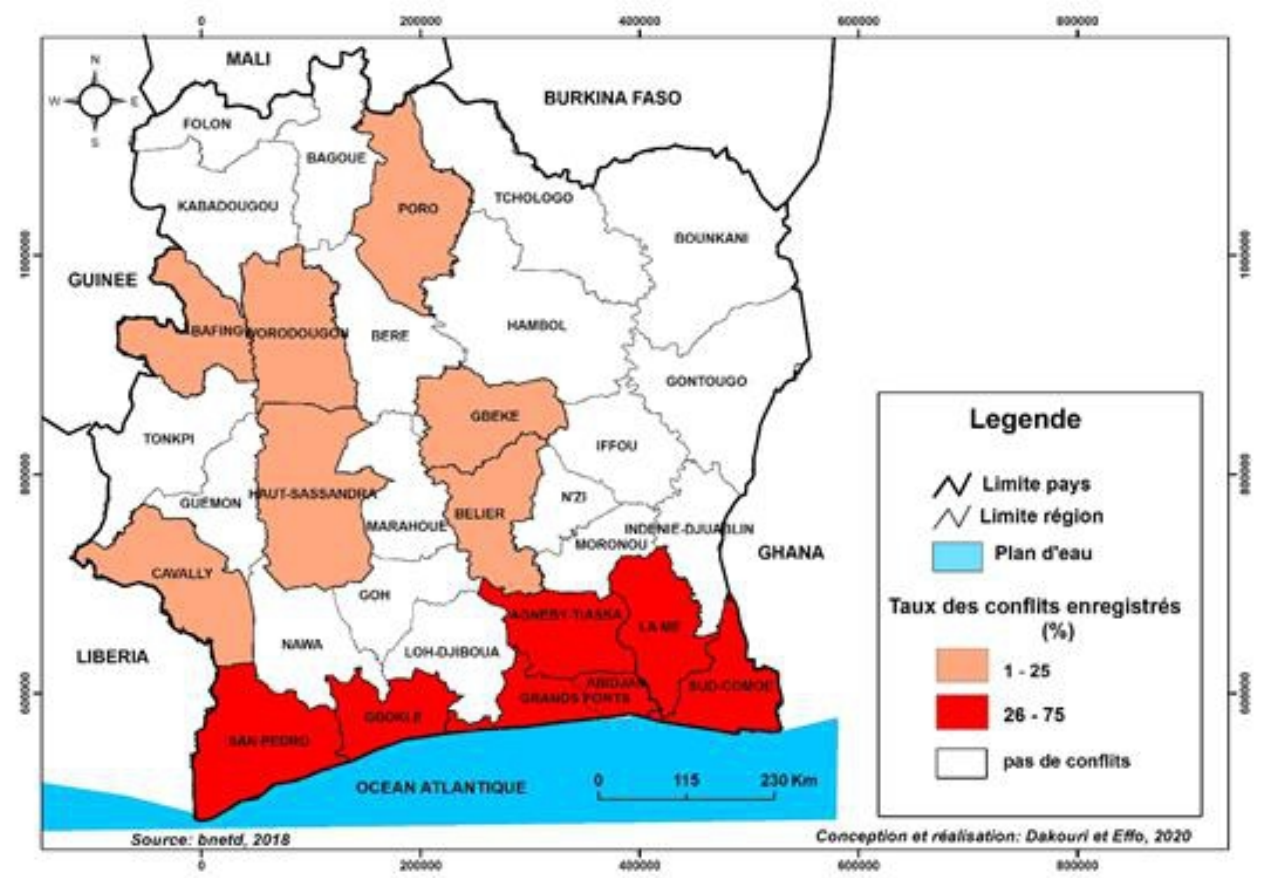

Figure 4. Intensité des conflits selon les régions

Mais c'est également un lieu de manifestation de conflits armés récurrents, conséquences des guerres civiles ayant affecté certains États ouestafricains (Libéria, Sierra Leone, Guinée, Mali, Côte d'Ivoire, Burkina Faso). C'est pourquoi, Kadet (2015, p.454) soutient que l'Ouest de la République de Côte d'Ivoire, est une zone grise, en raison des contradictions socio-spatiales qu'elle connait depuis plusieurs décennies.

\subsection{2- Au niveau des villes}

Depuis son accession à l'indépendance, plusieurs villes de la Côte d'Ivoire ont connu différentes sortes de conflits. Cependant, en ce qui concerne les conflits politiques, la ville d'Abidjan, capitale économique et chef-lieu de région du grand pont vient en tête et totalise à elle seule $70 \%$ des conflits comme le montre la Figure 5. Cela s'explique par la densité de la population et des activités économiques, la présence de toutes les communautés religieuses et groupes ethnoculturels. Cela s'explique également par la présence des sièges de tous les partis politiques et institutions de la république. La ville de Bouaké, capitale régionale du grand centre et deuxième ville du pays, arrive en seconde position 10\%, talonnées par Man la capitale régionale du grand ouest avec $8 \%$ et Korhogo, capitale régionale du grand nord qui enregistre 5\% des conflits. Les villes de San Pedro, Gagnoa et Abengourou comptent respectivement $3 \%, 2 \%$ et $2 \%$. 


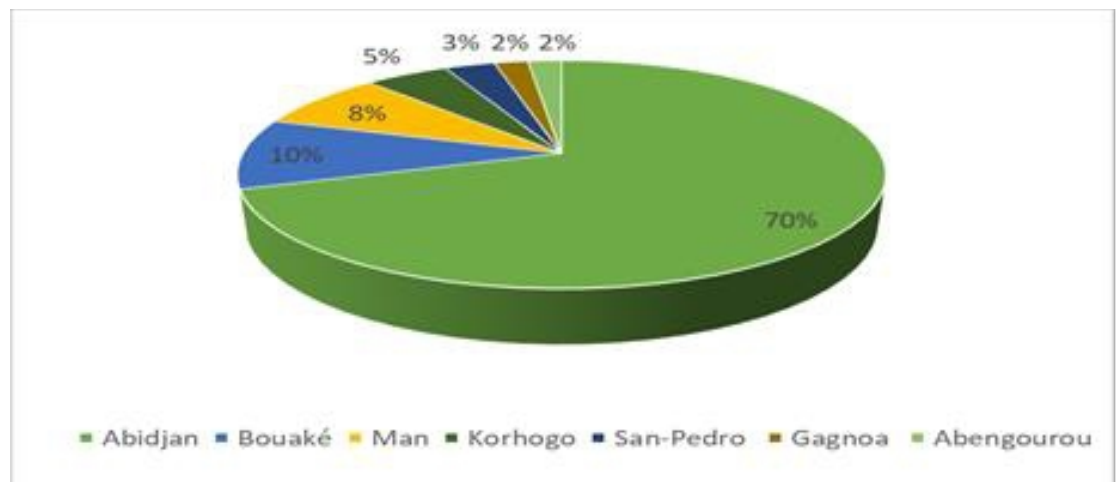

Figure 5. Répartition des conflits politiques selon les chef-lieux de région enquêtées.

Source : Nos enquêtes 2019

\section{4- La guerre de 2002 et la partition du pays en deux blocs antogonistes}

Pendant les 20 premières années de son indépendance, la Côte-d'Ivoire a connu une croissance économique exceptionnelle. On a même parlé du « miracle ivoirien ». Puis, dès le début des années 1980, le pays est entré dans une crise économique qui s'est par la suite muée en crise politique. Le 19 septembre 2002, des soldats déserteurs de l'armée ivoirienne, refugiés au Burkina Faso, aidés par des mercenaires, ont envahi la Côte-d'Ivoire. Ils sont arrivés jusqu'à Abidjan avant d'être repoussés. Ces hommes armés, occupèrent le nord, le centre et l'ouest du pays, soit $60 \%$ du territoire national (Figure 6).

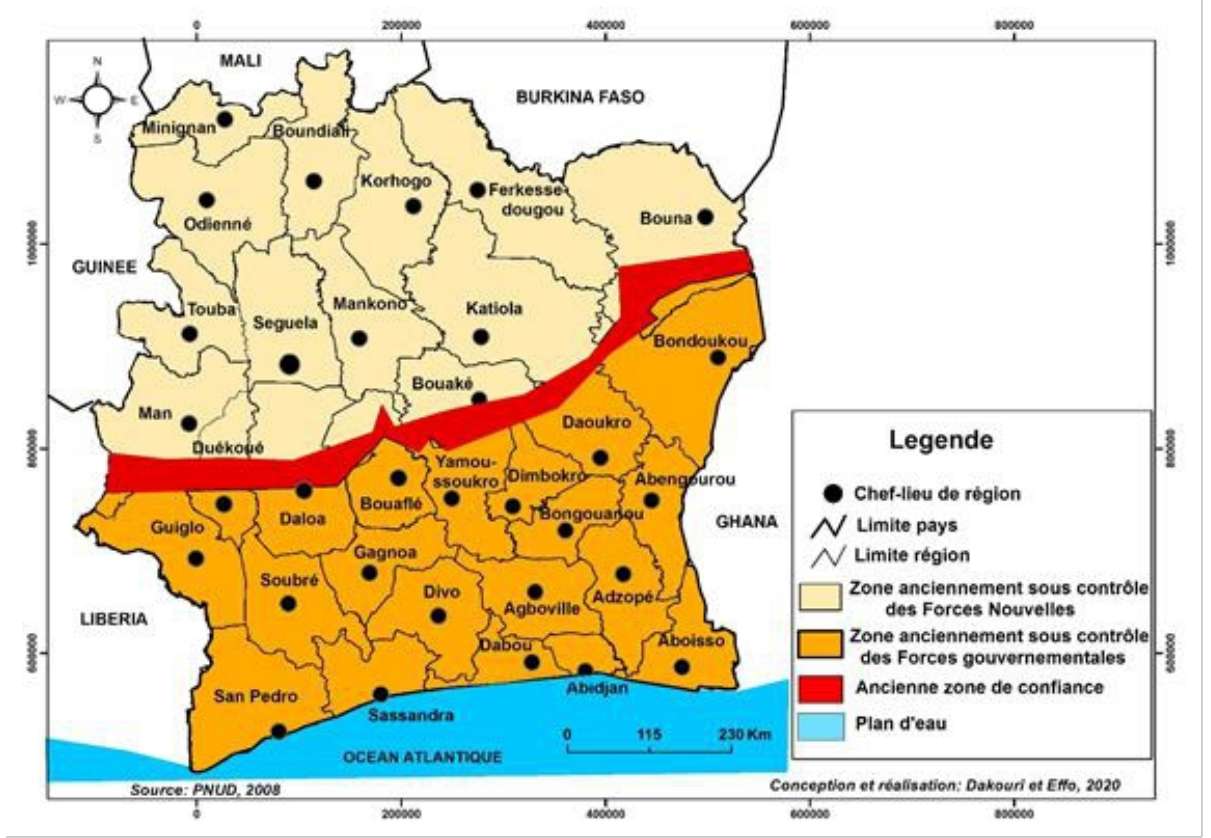

Figure 6. Vue de la Côte d'Ivoire divisée de 2002 à 2011 
Il a fallu huit ans de combats, de tractations et de négociations sous l'égide de la «Communauté internationale » conduite par la France, pour aboutir à l'organisation de l'élection présidentielle d'octobre et de novembre 2010. Cette élection présidentielle qui était censée mettre fin au conflit a, au contraire, exacerbé les passions et plongé le pays dans de terribles massacres. Le bilan est lourd et indique officiellement trois milles morts, des centaines de militaires et de civiles emprisonnés, des dizaines de milliers d'autres en exil. Des réponses ont été apportées à ces conflits politiques, et ont donné lieu à des tentatives de réconciliation.

\section{Conclusion}

Jadis vitrine de l'Afrique de l'ouest, la Côte d'Ivoire est aujourd'hui un pays à la recherche de ses repères. En effet, pendant les vingt premières années de son indépendance, le pays a connu une croissance économique exceptionnelle. On a même parlé du «miracle ivoirien ». Puis, dès le début des années 1980, le pays est entré dans une crise économique qui s'est par la suite muée en conflits politiques. Ces conflits ont gagné en intensité avec d'abord l'avènement du multipartisme, ensuite l'administration au pays des mesures d'austérité par la Banque Mondiale et le Fonds Monétaire International (FMI) et enfin la guerre des clans entre les héritiers du président Félix Houphouët Boigny dès sa disparition en 1993. Cette étude des conflits politiques sous l'angle spatial s'est efforcé d'abord d'identifier les facteurs de l'émergence de ces conflits avant de faire la cartographie des grands foyers de ceux-ci. En effet, si le tribalisme, la religion, la pauvreté, l'analphabétisme, la densité et l'extrême jeunesse de la population sont autant de facteurs de l'émergence des conflits politiques, c'est toute la zone sud jusqu'au littoral et les régions de grand pont Tonkpi, Haut-Sassandra, Bélier, Gbêkê, Bafing, Worodougou et Poro qui en constituent les grands foyers.

\section{References :}

1. Bamba, M. (2015). «Instabilité politique en Côte d'Ivoire de 1993 A 2011 ». Revue du CAMES, Sciences Humaines, Vol. 1, No 4, 16p.

2. Bonzon, S. (1967). "Modernisation et conflits tribaux en Afrique noire ». Revue française de science politique, $17^{\mathrm{e}}$ année, $\mathrm{n}^{\circ} 5$, pp. 862888.

3. Calas, B. (2011). «Introduction à une géographie des conflits... en Afrique ». Les Cahiers d'Outre-Mer, Revue de géographie de Bordeaux, n²55, pp. 295-320.

4. Canivez, P. (2008). Qu'est-ce qu'un conflit politique ? Revue de métaphysique et de morale, Presses Universitaires de France (2) $n^{\circ} 58$, pp. 163-175. 
5. Ebodé, N.V.J. (2006). « Les enfants soldats dans les crises africaines : entre logique militaire et stratégies politiques ». Presses Universitaires de France. Revue Guerres mondiales et conflits contemporains, vol.2, $\mathrm{n}^{\circ} 222$, pp.111-119.

6. Horace Mann (1883). De l'importance de l'éducation dans la république, Deuxième édition, revue et corrigée, (Français) Broché, édition Hachette Livre BNF, Collection : Sciences sociales, 90p.

7. Huntington, P.S. (1993). The clash of civilisations? Foreign Affairs, New Jersey, ABI/ INFORM Global, 22 p.

8. Joerin, F., Pelletier, M., Trudelle, C. \& Villeneuve, P. (2005). «Analyse spatiale des conflits urbains : enjeux et contextes dans la région de Québec ». Cahiers de géographie du Québec, vol. 49 n 138 , pp.319-342.

9. Kadet, G. B. (2015). «L'ouest forestier ivoirien : enjeux et problèmes d'une zone grise ». Les Cahiers d'Outre-Mer, n'271, pp. 285-462.

10. Koui, T. (2010). La côte d'ivoire, les cavaliers de l'apocalypse, Paris, Edition Harmattan, 87p.

11. Lecourt, A. \& Baudelle, G. (2004). Confl its d'aménagement et proximité sociale: une réévaluation. International Journal of Sustainable Development, vol. 7, no 3, pp. 287-301.

12. Lonsdale, J. (2003). "Le cas kenyan : un débat moral et politique". Politique africaine, $\mathrm{n}^{\circ}$ 90, juin, p. 17-36.

13. ONU (2004). Guide du protocole facultatif concernant l'implication d'enfants dans les conflits armés. Coalition contre l'utilisation d'enfants soldats, UNICEF, $74 \mathrm{p}$.

14. Retaillé, D. (2011). «Introduction à une géographie des conflits » L'Information géographique, Armand Colin (3) Vol. 75, pp. 6-22

15. Soriano, C.P. (2001). Eduquer dans et pour le conflit, Chaire Unesco pour la paix et les droits de l'homme, 32p. 